\title{
Classical dimensional transmutation and confinement
}

\author{
Gia Dvali, ${ }^{a, b, d, c}$ Cesar Gomez $^{e}$ and Slava Mukhanov ${ }^{a, b}$ \\ ${ }^{a}$ ASC, Department für Physik, LMU, München, \\ Theresienstr. 3\%, 80333 München, Germany \\ ${ }^{b}$ MPI für Physik, \\ Föhringer Ring 6, 80805 München, Germany \\ ${ }^{c}$ CERN, Theory Division, \\ 1211 Geneva 23, Switzerland \\ ${ }^{d}$ CCPP, Department of Physics, NYU, \\ 4 Washington Place, New York, NY 10003, U.S.A. \\ ${ }^{e}$ Instituto de Física Teórica UAM-CSIC, C-XVI, Universidad Autónoma de Madrid, \\ Cantoblanco, 28049 Madrid, Spain \\ E-mail: Georgi.Dvali@cern.ch, bfkl08@gmail.com, mukhanov@physik.lmu.de
}

ABSTRACT: We observe that probing certain classical field theories by external sources uncovers the underlying renormalization group structure, including the phenomenon of dimensional transmutation, at purely-classical level. We perform this study on an example of $\lambda \phi^{4}$ theory and unravel asymptotic freedom and triviality for negative and positives signs of $\lambda$ respectively. We derive exact classical $\beta$ function equation. Solving this equation we find that an isolated source has an infinite energy and therefore cannot exist as an asymptotic state. On the other hand a dipole, built out of two opposite charges, has finite positive energy. At large separation the interaction potential between these two charges grows indefinitely as a distance in power one third.

Keywords: Asymptotic Freedom, Renormalization Group, Renormalization Regularization and Renormalons

ArXiv EPRINT: 1107.0870 


\section{Contents}

1 Introduction 1

2 Classical solution $\quad 2$

2.1 Anti-screening 2

2.2 The perturbative expansion 3

3 Renormalization group and asymptotic freedom 5

$\begin{array}{ll}3.1 & \text { Perturbative } \beta \text { function }\end{array}$

3.2 Partial resummations 8

$\begin{array}{lll}3.3 & \text { Dimensional transmutation and asymptotic freedom } & 10\end{array}$

4 Beyond perturbation theory and asymptotic behavior $\quad 10$

4.1 Exact classical $\beta$ function 11

$\begin{array}{lll}4.2 & \text { Weak coupling expansion and renormalons } & 12\end{array}$

5 The nonperturbative solution and confinement $\quad 13$

$\begin{array}{ll}5.1 & \text { The infrared coupling constant } \\ 5.2 & 13\end{array}$

$\begin{array}{lll}5.2 \text { Confinement } & 15\end{array}$

$\begin{array}{lll}6 & \text { Discussion and speculations } & 17\end{array}$

$\begin{array}{ll}\text { A On the triviality of } \lambda \phi^{4} \text { theory with positive } \lambda & 17\end{array}$

$\begin{array}{lr}\text { B Confinement with and without strings } & 19\end{array}$

\section{Introduction}

The discovery of asymptotic freedom in QCD [1, 2] opened a new era in particle physics. Besides its direct relevance for understanding the nature of strong interactions, it introduced a concept of dimensional transmutation or equivalently a dynamical generation of scale in a seemingly scale-free theory. Both phenomena, asymptotic freedom and dimensional transmutation, are usually perceived as intrinsically quantum phenomena, as they are both deeply rooted in the renormalization group properties of the quantum theory. The question we would like to address in this paper is, how much of these phenomena is captured by classical physics? This is a fully legitimate question, since usually quantum effects have classical precursors, which sometimes appear in the form of uncontrollable growth of the classical fields.

In order to illustrate our ideas, we will consider a simple example, namely, scalar theory with negative $\lambda \phi^{4}$, which is known to be renormalizable and has a negative $\beta$ function $[3,4]$, 
but has an unbounded from below potential. However, our classical renormalization group treatment delivers a natural prescription which allows to self-consistently work with this theory in the presence of external sources, and isolate our findings from the issue of potential instability in a pure $\lambda \phi^{4}$ theory.

In the presence of the external sources, the requirement of independence of physical observable from the regulator scale of sources implies the running of the effective coupling. This running is the main reason behind the whole renormalization group structure and the subsequent classical dimensional transmutation. This scale dependence is the key to why the renormalization group results can be safely disentangled from the instability issues. Simply speaking, because of the emerging scale-dependence we always perform calculations on time-scales shorter than would-be instability time in a sourceless theory.

Surprisingly, by probing $\lambda \phi^{4}$ theory by the large external source, we uncover the whole built-in RG structure already at the classical level, with fully-fledged counterparts of asymptotic freedom as well as dimensional transmutation phenomena, in which an analog of QCD-scale appears as a result of classical $R G$ invariance. We derive the exact classical $\beta$ function equation from which we extract non perturbative information about the infrared region. Solving this equation in strong coupling regime we find that the energy of the isolated external charge is infinite and positive and hence it cannot exist as a free asymptotic state. Moreover, considering a dipole, build out of two charges with opposite signs, we find that its energy is positive and finite. When charges in this dipole are separated their interaction potential grows indefinitely as distance in power one third, thus confining the charges. These findings indicate that there may exist a classical counterpart of confinement.

\section{Classical solution}

After the discovery of asymptotic freedom in the non-Abelian Yang Mills theories, the physics underlying the negative sign of the $\beta$ function was understood as an anti-screening effect due to self-interactions of the gauge fields. From the classical point of view we can try to understand this anti-screening considering how self-interaction modifies the field created by an external point-like source $Q$ at large distances. This modification compared to the case of free fields can be used to define the effective charge $Q_{\text {eff }}(r)$ at distance $r$ or, equivalently, the running coupling constant $\alpha(r)$. This can be done without invoking quantum theory and the result will only depend on the particular classical features of selfinteractions. For the large external charge one can expect that the classical contribution to anti-screening will dominate over the one due to the vacuum polarization effects.

In this section we will solve perturbatively the classical equations of motion for a given external charge and show how the anti-screening effect (the growth of $Q_{\text {eff }}(r)$ with $r$ ) can naturally be achieved.

\subsection{Anti-screening}

Let us consider $\lambda_{0} \phi^{4}$ theory with negative $\lambda_{0}$

$$
S=\int\left(\frac{1}{2} \partial_{\mu} \phi \partial^{\mu} \phi-\frac{1}{4} \lambda_{0} \phi^{4}+4 \pi Q \phi\right) d^{4} x
$$


where the signature is taken to be,+--- , and $Q$ is the external charge. In the case of a point-like charge the field equation for the static spherically symmetric field $\phi(r)$ reduces to

$$
\frac{1}{r^{2}} \frac{d}{d r}\left(r^{2} \frac{d \phi}{d r}\right)-\lambda_{0} \phi^{3}=-4 \pi Q \delta(\mathbf{x}) .
$$

If one neglects the nonlinear term in this equation then its solution is

$$
\phi_{0}=\frac{Q}{r} .
$$

Notice that for $Q \gg 1$ the amplitude of the classical scalar field on scales $r$ is much larger than the typical amplitude of the quantum fluctuations, which is of order $1 / r$. If the coupling constant $\lambda_{0}$ is small enough, i.e. $\lambda_{0} Q^{2} \ll 1$, then the corrections to solution (2.2) due to the self-interaction $\lambda_{0} \phi^{3}$ can be treated perturbatively. The leading order correction to the solution $\phi_{0}$ can be obtained by solving equation

$$
\frac{1}{r^{2}} \frac{d}{d r}\left(r^{2} \frac{d \phi}{d r}\right)=-4 \pi Q \delta(\mathbf{x})+\lambda_{0} \frac{Q^{3}}{r^{3}}
$$

where we have substituted $\phi_{0}$ in the nonlinear term. The last term in this equation can be treated as the contribution to the effective charge induced by the nonlinear self-interaction. As it was noticed above, for $Q \gg 1$, the vacuum polarization contribution to the induced charge is much smaller than the classical contribution and therefore can be neglected. As one can easily see from (2.4) the induced charge for positive $\lambda_{0}$ has a sign which is opposite to the sign of the source and the nonlinear interaction leads to screening. For negative $\lambda_{0}$ the charges have the same sign and we have an anti-screening effect similar to the one of the non-Abelian gauge theories. Since we are mainly interested in asymptotic freedom and confinement we will consider only the case of negative $\lambda_{0}$.

\subsection{The perturbative expansion}

Let us look for the solution to equation (2.2) in the following form

$$
\phi(r)=\frac{Q f(r)}{r} .
$$

Integrating equation (2.2) and substituting this ansatz we can rewrite the equation for the scalar field as

$$
f(r)=1+\alpha_{0} r \int_{r}^{\infty}\left(\int_{r_{0}}^{r^{\prime}} \frac{f^{3}\left(r^{\prime \prime}\right)}{r^{\prime \prime}} d r^{\prime \prime}\right) \frac{d r^{\prime}}{r^{\prime 2}}-N\left(\alpha_{0}\right),
$$

where

$$
\alpha_{0} \equiv-\lambda_{0} Q^{2}>0
$$

is the effective coupling constant and we have introduced the ultraviolet cutoff scale $r_{0}$ to regularize the integral, which otherwise would diverge. The function $N\left(\alpha_{0}\right)$, which depends only on $\alpha_{0}$, is fixed by the normalization condition: $f\left(r_{0}\right)=1$. It is clear that in the limit $\alpha_{0} \rightarrow 0$ it must vanish and therefore in the absence of self-interaction, the solution (2.5) with $f(r)=1$ exactly satisfies equation (2.2). As it follows from (2.5), the 
function $f$ defines the anti-screened effective charge $Q_{\text {eff }}(r)=Q f(r)$ or, equivalently, the running coupling constant

$$
\alpha_{\text {eff }}(r)=\alpha_{0} f^{2}(r)
$$

Assuming that $\alpha_{0} \ll 1$ we can solve the integral equation (2.7) by iterations in powers of $\alpha_{0}$. With this purpose it is convenient to rewrite it as

$$
f(x)=1+\alpha_{0} e^{x} \int_{x}^{\infty}\left(\int_{0}^{x^{\prime}} f^{3}\left(x^{\prime \prime}\right) d x^{\prime \prime}\right) e^{-x^{\prime}} d x^{\prime}-N\left(\alpha_{0}\right),
$$

where we have introduced $x=\ln \left(r / r_{0}\right)$ instead of $r$. Substituting $f(x)=1$ into the right hand side of equation (2.9) and taking into account that $N\left(\alpha_{0}\right)=\alpha_{0}+O\left(\alpha_{0}^{2}\right)$ we find

$$
f(x)=1+\alpha_{0} x+O\left(\alpha_{0}^{2}\right) .
$$

Next we take this solution, substitute it again in (2.9) and take $N\left(\alpha_{0}\right)=\alpha_{0}+3 \alpha_{0}^{2}+O\left(\alpha_{0}^{3}\right)$. Keeping only the terms up to second order in $\alpha_{0}^{2}$ leads to

$$
f(x)=1+\alpha_{0} x+\alpha_{0}^{2}\left(\frac{3}{2} x^{2}+3 x\right)+O\left(\alpha_{0}^{3}\right) .
$$

This procedure can be iterated giving us at each step the next order term in $\alpha_{0}$. The result up to order $\alpha_{0}^{6}$ is

$$
\begin{aligned}
f(x)=1 & +\alpha_{0} x+\alpha_{0}^{2}\left(\frac{3}{2} x^{2}+3 x\right)+\alpha_{0}^{3}\left(\frac{5}{2} x^{3}+12 x^{2}+24 x\right) \\
& +\alpha_{0}^{4}\left(\frac{35}{8} x^{4}+\frac{71}{2} x^{3}+\frac{285}{2} x^{2}+285 x\right) \\
& +\alpha_{0}^{5}\left(\frac{63}{8} x^{5}+93 x^{4}+\frac{1143}{2} x^{3}+2142 x^{2}+4284 x\right) \\
& +\alpha_{0}^{6}\left(\frac{231}{16} x^{6}+\frac{9129}{40} x^{5}+\frac{7665}{4} x^{4}+10521 x^{3}+37989 x^{2}+75978 x\right)+O\left(\alpha_{0}^{7}\right) .
\end{aligned}
$$

The function $N\left(\alpha_{0}\right)$ to the same order in perturbations should be taken to be

$$
N\left(\alpha_{0}\right)=\alpha_{0}+3 \alpha_{0}^{2}+24 \alpha_{0}^{3}+285 \alpha_{0}^{4}+4284 \alpha_{0}^{5}+75978 \alpha_{0}^{6}+O\left(\alpha_{0}^{7}\right) .
$$

The effective running coupling as a function of distance can be written then as perturbative series in powers of $\alpha_{0}$

$$
\alpha_{\mathrm{eff}}(x)=\alpha_{\mathrm{eff}}(r)=\alpha_{0} f^{2}(r)=\sum_{n=0}^{\infty} \alpha_{0}^{n+1} g_{n}(x),
$$

where

$$
\begin{aligned}
& g_{0}(x)=1, g_{1}(x)=2 x, g_{2}(x)=4 x^{2}+6 x, g_{3}(x)=8 x^{3}+30 x^{2}+48 x, \\
& g_{4}(x)=16 x^{4}+104 x^{3}+342 x^{2}+570 x,
\end{aligned}
$$




$$
\begin{aligned}
& g_{5}(x)=32 x^{5}+308 x^{4}+1572 x^{3}+4998 x^{2}+8568 x, \\
& g_{6}(x)=64 x^{6}+\frac{4176}{5} x^{5}+5880 x^{4}+27612 x^{3}+86832 x^{2}+151956 x,
\end{aligned}
$$

etc. The calculation of $g_{n}(x)$ is straightforward and we did it until $g_{10}(x)$. However, to simplify the formulae we present here the result only up to $g_{6}(x)$. Note that the running coupling constant depends on $r$ only logarithmically with the coefficients $g_{n}$ power series of $x=\ln \left(r / r_{0}\right)$ with the highest power $n$. Moreover, the series (2.14) can be rearranged and partially resummed. In particular, collecting together leading powers of logarithms, next-to-leading and next-to-next leading powers we get

$$
\begin{aligned}
\alpha_{\mathrm{eff}}(x)= & \alpha_{0}\left[1+2 \tilde{x}+4 \tilde{x}^{2}+8 \tilde{x}^{4}+16 \tilde{x}^{5}+32 \tilde{x}^{6}+O\left(\tilde{x}^{7}\right)\right] \\
& +\alpha_{0}^{2}\left[6 \tilde{x}+30 \tilde{x}^{2}+104 \tilde{x}^{3}+308 \tilde{x}^{4}+\frac{4176}{5} \tilde{x}^{5}+\frac{10704}{5} \tilde{x}^{6}+O\left(\tilde{x}^{7}\right)\right] \\
& +\alpha_{0}^{3}\left[48 \tilde{x}+342 \tilde{x}^{2}+1572 \tilde{x}^{3}+5880 \tilde{x}^{4}+\frac{97248}{5} \tilde{x}^{5}+59248 \tilde{x}^{6}+O\left(\tilde{x}^{7}\right)\right] \\
& +O\left(\alpha_{0}^{4} \ldots\right)
\end{aligned}
$$

where $\tilde{x}=\alpha_{0} x$. In the second and third brackets we have also included higher order terms compared to (2.15). The series in the bracket can be resummed. In particular, it is obvious that

$$
1+2 \tilde{x}+4 \tilde{x}^{2}+8 \tilde{x}^{4}+16 \tilde{x}^{5}+32 \tilde{x}^{6}+\ldots=\frac{1}{1-2 \tilde{x}},
$$

Much less obvious are the following results

$$
6 \tilde{x}+30 \tilde{x}^{2}+104 \tilde{x}^{3}+308 \tilde{x}^{4}+\frac{4176}{5} \tilde{x}^{5}+\ldots=\frac{3 \ln (1-2 \tilde{x})}{(1-2 \tilde{x})^{2}}
$$

and

$$
\begin{aligned}
& 48 \tilde{x}+342 \tilde{x}^{2}+1572 \tilde{x}^{3}+5880 \tilde{x}^{4}+\frac{97248}{5} \tilde{x}^{5}+59248 \tilde{x}^{6} \ldots \\
& =\frac{9(\ln (1-2 \tilde{x}))^{2}-9 \ln (1-2 \tilde{x})+30 \tilde{x}}{(1-2 \tilde{x})^{3}}
\end{aligned}
$$

which the reader can verify just expanding the appropriate expressions in powers of $\tilde{x}$. One may wonder how did we manage to resum these last two series? The answer to this question is in the next section where we uncover the renormalization group structure of our entirely classical theory and derive the $\beta$ function which generates the resummation of the perturbative expansion to the appropriate powers of $\alpha_{0}$.

\section{Renormalization group and asymptotic freedom}

In the Wilsonian approach [5-8] the renormalization group sets how the couplings of the quantum theory should change under re-scalings of the UV cutoff . The equations governing this dependence are known as the renormalization group equations. This general notion of renormalization group can be extended to the classical field theory with external 
point-like sources in the following sense. Let us introduce an UV cutoff $r_{0}$ setting the way we smear the source. The classical field created by such source will generically depends on the regulator $r_{0}$ and the self-coupling $\lambda$ of the theory. One can ask under which circumstances we can require that the classical theory must be invariant under re-scaling of $r_{0}$. This is possible only if the corresponding classical theory incorporates the renormalization group structure. In this case the dependence of the coupling on the smearing cutoff $\lambda\left(r_{0}\right)$ also captures the screening and anti-screening effects. Moreover, using the effective running coupling we can associate with an external source, a physical length scale $R_{c}$ by the standard procedure of dimensional transmutation.

The reason why the classical solution captures the renormalization group structure is easy to understand. Any regularization scheme in quantum field theory give rise to logarithmic contributions which even in a scale invariant theory lead to anomalous scaling. These logarithmic contributions are of the type $\log (p / \Lambda)$ with $\Lambda$ the UV cutoff. Since the divergent contribution $\log \Lambda$ is absorbed by renormalization, we are free to choose the scale $p$ at which the logarithmic contribution to the self-energy vanishes. As a consequence the scaling of $\Lambda$ should be accompanied by finite renormalizations (the RG transformations) of the coupling constants. In the classical theory under consideration we have found the same type of logarithmic contributions to the field created by the external source. In this case the role of cutoff $\Lambda$ is played by the smearing scale $r_{0}$. One can renormalize the classical theory by subtracting the $\log r_{0}$ contributions as it is done in quantum field theory. However, if we want physics to be independent on the method of removing this infinity, we need to change the couplings, exactly as it is done in quantum field theory. Both renormalization group structures, the classical and the quantum ones, are structurally identical for $\lambda \phi^{4}$ theory because both have the same type of parent logarithmic contributions.

\subsection{Perturbative $\beta$ function}

Once we have obtained the classical expression (2.14) for the effective coupling constant $\alpha_{\text {eff }}(r)$ we can check whether taking the bare coupling constant $\alpha_{0}$ as a function of $r_{0}$ we can make $\alpha_{\text {eff }}(r)$ independent of $r_{0}$. As we have said, this is possible only in the theories with associated renormalization group structure, which in turn imposes rather severe conditions on the functions $g_{n}(x)$ in the perturbative expansion (2.14). Let us first derive these conditions, which do not depend on the origin (classical or quantum) of the renormalization group, and then verify whether they are satisfied by the functions in (2.15).

On general grounds the expansion of the dimensionless running coupling constant $\alpha_{\text {eff }}(r)$ in powers of $\alpha_{0}=\alpha\left(r_{0}\right)$, normalized at $r=r_{0}$, can be written as

$$
\alpha_{\mathrm{eff}}(r)=\alpha\left(r_{0}\right)+\alpha^{2}\left(r_{0}\right) g_{1}\left(\frac{r}{r_{0}}\right)+\ldots=\sum_{n=0}^{\infty} \alpha^{n+1}\left(r_{0}\right) g_{n}\left(\frac{r}{r_{0}}\right)
$$

where we use the spatial scale $r$ instead of the usually used energy scale $k \sim 1 / r$. It is clear that $g_{0}\left(r / r_{0}\right)=1$ and since $\alpha(r)=\alpha\left(r_{0}\right)$ at $r=r_{0}$, we have

$$
g_{n}(1)=0,
$$


for $n \geq 1$. Invariance under changes of the ultraviolet regulator $r_{0}$, implies

$$
\frac{d}{d r_{0}}\left(\sum_{n=0}^{\infty} \alpha^{n+1}\left(r_{0}\right) g_{n}\left(\frac{r}{r_{0}}\right)\right)=0,
$$

which in turn imposes severe restrictions on $g_{n}\left(r / r_{0}\right)$. Taking the derivative and rearranging the terms in (3.3) leads to

$$
\frac{d \alpha\left(r_{0}\right)}{d \ln r_{0}}=\alpha^{2}\left(r_{0}\right) \frac{\sum_{k=0}^{\infty} g_{k+1}^{\prime}(x) \alpha^{k}\left(r_{0}\right)}{\sum_{k=0}^{\infty}(k+1) g_{k}(x) \alpha^{k}\left(r_{0}\right)},
$$

where $x=\ln \left(r / r_{0}\right)$ and prime denotes the derivative with respect to $x$. The ratio of sums in the right hand side of (3.4) should not depend on $x$ because the left hand side of this equation is $x$-independent. Therefore setting $x=0$ (which corresponds to $r=r_{0}$ ) and taking into account (3.2) we find that

$$
\frac{\sum_{k=0}^{\infty} g_{k+1}^{\prime}(x) \alpha^{k}\left(r_{0}\right)}{\sum_{k=0}^{\infty}(k+1) g_{k}(x) \alpha^{k}\left(r_{0}\right)}=\sum_{k=0}^{\infty} g_{k+1}^{\prime}(0) \alpha^{k}\left(r_{0}\right)
$$

from where it follows that the functions $g_{k}(x)$ should satisfy the following recursion relations:

$$
\frac{d g_{n+1}(x)}{d x}=\sum_{k=0}^{n}(k+1) g_{n+1-k}^{\prime}(0) g_{k}(x) .
$$

Note that only if these conditions are satisfied then there exists a function $\alpha\left(r_{0}\right)$ for which the sum in the right hand side of (3.1) does not depend on $r_{0}$. Nicely enough the unambiguous solution of these recursion relations with "initial conditions" (3.2) is given by

$$
g_{n}(x)=\sum_{k=1}^{n} c_{k} x^{k}
$$

where $c_{k}$ are completely determined by the numerical values of $g_{1}^{\prime}(0), g_{2}^{\prime}(0), \ldots$ which in principle can be arbitrary. For instance, the coefficient in front of the leading logarithm $x^{n}=\ln ^{n}\left(r / r_{0}\right)$ in $g_{n}$ is equal to $c_{n}=\left(g_{1}^{\prime}(0)\right)^{n}$.

At this point it is quite rewarding to confirm that the set of classical functions (2.15) in fact satisfies the recursion relations (3.6). This can be done by direct calculation to any order in perturbation theory (we did it up to $g_{10}$ ). Thus, taking $\alpha_{0}$ in (2.14) to be the function of $r_{0}$ we uncover the renormalization group structure of the classical $\lambda \phi^{4}$ theory. We would like to stress that in distinction from the quantum field theory, where the renormalization group is normally checked by direct calculations only to the leading logarithms (and postulated otherwise), we verified it also for all subleading logarithms. 
To take the advantage of renormalization group for partial resummation of the perturbative expansion (2.14) we note that from (3.4) and (3.5) it follows

$$
\frac{d \alpha\left(r_{0}\right)}{d \ln r_{0}}=\alpha^{2}\left(r_{0}\right) \sum_{k=0}^{\infty} g_{k+1}^{\prime}(0) \alpha^{k}\left(r_{0}\right) .
$$

The running constant $\alpha_{\text {eff }}(r)$ depends on $r$ in the same way that $\alpha\left(r_{0}\right)$ depends on $r_{0}$. Hence $\alpha_{\text {eff }}(x)$ satisfies the well known Gell-Mann-Low equation [9]

$$
\frac{d \alpha_{\mathrm{eff}}(x)}{d x}=\alpha_{\mathrm{eff}}^{2}(x) \sum_{k=0}^{\infty} g_{k+1}^{\prime}(0) \alpha_{\mathrm{eff}}^{k}(x) .
$$

The $\beta$ function is normally defined as the derivative of $\alpha_{\text {eff }}$ with respect to the logarithm of the energy squared. For us it is more convenient to define it as

$$
\beta \equiv \frac{d \alpha_{\mathrm{eff}}(x)}{d x},
$$

which (up to factors $4 \pi$ due to the choice of charge units) is related to the standard $\beta_{s t}$ function as $\beta_{s t}=-\beta / 2$. According to (3.9) and (2.15) the classical perturbative $\beta$ function is equal to

$$
\begin{aligned}
\beta(\alpha)=\sum_{k=1}^{\infty} \beta_{k} \alpha^{k+1}= & 2 \alpha^{2}+6 \alpha^{3}+48 \alpha^{4}+570 \alpha^{5} \\
& +8568 \alpha^{6}+151956 \alpha^{7}+\ldots,
\end{aligned}
$$

where $\alpha \equiv \alpha_{\text {eff }}(x)$ and $\beta_{i} \equiv g_{i}^{\prime}(0)$.

Obviously, we should not expect the numerical coefficients $\beta_{i}$ of this classical beta function to be identical to the ones derived in the quantum field theory. In the last case $\beta_{i}$ are determined by the loop contributions and they will depend, beyond two loops, on the particular renormalization scheme used to segregate a finite part of the divergent loop integrals. The classical beta function accounts for the anti-screening effects due to the classical self-interaction. A potential quantum theory check of the numerical coefficients derived above will require to work in the presence of large external charge where we have to modify the Green functions in order to account for the effect of the external charge. Because for $Q \gg 1$ the quantum fluctuations are subdominant we expect that classical contribution dominates. Although the direct check of this expectation is obviously important we will not follow that path. Instead we will restrict ourselves to the physical consequences of the underlying renormalization group structure of the classical theory.

\subsection{Partial resummations}

In equation (2.16) we have separately collected the contribution of the leading and subleading logarithms to $\alpha(x)$ and presented the result of their resummation. For the subleading logarithm the result was derived using Gell-Mann-Low equation. For $\alpha \ll 1$ we can first 
neglect all terms in $\beta$ function besides of the "one loop" contribution. Equation (3.9) then reduces to

$$
\frac{d \alpha(x)}{d x}=2 \alpha^{2}(x),
$$

and its solution, with initial condition $\alpha(0)=\alpha_{0} \equiv-\lambda_{0} Q^{2}$, is

$$
\alpha(r)=\frac{\alpha_{0}}{1-2 \alpha_{0} x}=\frac{-\lambda_{0} Q^{2}}{1+2 \lambda_{0} Q^{2} \ln \left(r / r_{0}\right)},
$$

where $\lambda_{0}=\lambda\left(r_{0}\right)<0$. It is easy to see that this solution gives us the resummation of the leading logarithms in the expansion (2.16), (2.17).

We can repeat the same analysis keeping in Gell-Mann-Low equation the contribution up to two loops,

$$
\frac{d \alpha(x)}{d x}=2 \alpha^{2}(x)+6 \alpha^{3} .
$$

Integrating this equation with initial condition $\alpha(0)=\alpha_{0}$, we obtain

$$
\frac{1}{\alpha(x)}-3 \ln \left(\frac{1+3 \alpha(x)}{1+3 \alpha_{0}} \times \frac{\alpha_{0}}{\alpha(x)}\right)=\frac{1-2 \alpha_{0} x}{\alpha_{0}} .
$$

Solving this equation in terms of the perturbative expansion in $\alpha_{0}$ one gets

$$
\alpha(x)=\frac{\alpha_{0}}{1-2 \alpha_{0} x}-3\left(\frac{\alpha_{0}}{1-2 \alpha_{0} x}\right)^{2} \ln \left(1-2 \alpha_{0} x\right)+O\left(\alpha_{0}^{3}\right)
$$

Note that the second term agrees with resummation (2.18) of the next to the leading order logarithms. The same is true at three loop order, where the solution to

$$
\frac{d \alpha(x)}{d x}=2 \alpha^{2}(x)+6 \alpha^{3}+48 \alpha^{4}
$$

which is

$$
\begin{aligned}
\alpha(x)= & \frac{\alpha_{0}}{1-2 \alpha_{0} x}-3\left(\frac{\alpha_{0}}{1-2 \alpha_{0} x}\right)^{2} \ln \left(1-2 \alpha_{0} x\right) \\
& +9\left(\frac{\alpha_{0}}{1-2 \alpha_{0} x}\right)^{3}\left(\ln ^{2}\left(1-2 \alpha_{0} x\right)-\ln \left(1-2 \alpha_{0} x\right)+\frac{30}{9} \alpha_{0} x\right)+O\left(\alpha_{0}^{4}\right),
\end{aligned}
$$

also accounts for the resummation of next-to-next subleading logarithms. In other words the solutions to the classical renormalization group equation give us the resummation of the perturbative series (2.14) taking care in every step about next logarithms in $g_{n}(x)$. It is clear that when the running coupling constant becomes of order unity (strong coupling regime) all terms in expansion (3.18) are of the same order and the series (3.18) should be further resummed. It is not a priori clear whether the singularity in this expansion (Landau pole [10-12]) will survive after this resummation. We will answer this question in the next section using nonperturbative methods. 


\subsection{Dimensional transmutation and asymptotic freedom}

One important consequence of the renormalization group is dimensional transmutation. We can easily understand this phenomenon using the result of the one loop resummation of perturbative expansion

$$
\alpha(r)=\frac{-\lambda_{0} Q^{2}}{1+2 \lambda_{0} Q^{2} \ln \left(r / r_{0}\right)} .
$$

In this expression $\lambda_{0}$ depends on the regulator $r_{0}$ in such a way that $\alpha(r)$ is $r_{0}$-independent to the corresponding order. Therefore we can define the renormalization group invariant physical scale $R_{c}$ via

$$
\ln \frac{R_{c}}{r_{0}}=-\frac{1}{2 \lambda_{0} Q^{2}}
$$

Note that this scale

$$
R_{c}=r_{0} e^{-\frac{1}{2 \lambda\left(r_{0}\right) Q^{2}}}
$$

does not depend on the particular value of regulator $r_{0}$ at one loop level. Using this dynamically generated scale we can rewrite the physical running coupling as

$$
\alpha(r) \equiv-\lambda(r) Q^{2}=\frac{1}{2 \ln \left(R_{c} / r\right)} .
$$

The physical meaning of this expression is obvious. Perturbatively the theory can be defined in the ultraviolet region corresponding to length scales $r \ll R_{c}$, where it becomes effectively free. Thus, we have found asymptotic freedom in the classical $\lambda \phi^{4}$ theory with negative $\lambda$.

In the infrared at length scales of order $R_{c}$ the theory becomes strongly coupled and non-perturbative. What is the potential meaning of this dynamically generated scale? From the point of view of the classical theory the existence of this scale is quite surprising since it is independent of the UV regulator. On the top of that $R_{c}$ is a very non-perturbative scale. Obviously it is tempting to think of $R_{c}$ as setting the natural confinement scale of the theory. A way to check this claim is to derive an exact classical $\beta$ function equation and to read off the previous perturbative expansion from the corresponding solution of this equation. We address these issues below.

\section{Beyond perturbation theory and asymptotic behavior}

The usual way to address the non-perturbative phenomena within perturbation theory is to study the convergence of the perturbative series. In [13-16] it was found that the numerical coefficients in the perturbative expansion of $\beta$ function asymptotically grow as $\beta_{k} \sim k ! \beta_{1}^{k}$, where $\beta_{1}$ is one loop $\beta$ function and $k$ denotes the perturbative order in coupling constant. Such behavior sets the limit of perturbation theory and fixes the uncertainty of the computations to be of order $\exp \left(-1 / \beta_{1} \alpha\right)$. In the theories with asymptotic freedom this uncertainty is extremely small in the deep UV region contrary to what happens in the theories with UV Landau pole. Normally in quantum field theory is hard to prove this factorial asymptotic behavior of the coefficients in $\beta$ function. It can have different origin: either the growth of the number of diagrams contributing to a given order in perturbation theory (instanton effect) or the contribution of multi-bubble diagrams (renormalons). 
In the classical approach to the renormalization group, however, there is an opportunity to convert the classical equations of motion into exact equation for the $\beta$ function. This equation be can used afterwards to check the similarity between classical and quantum renormalization groups. In particular, as we will see, one can use the exact equation to derive the asymptotic behavior of the coefficients in the perturbative expansion of $\beta$ function. Interestingly enough the asymptotic behavior anticipated by the exact classical $\beta$ function agrees with the quantum filed theory expectations. In addition this allows us to clarify the origin of renormalons as well as the generic form of the non-perturbative uncertainties. Moreover, the non-perturbative contributions will be naturally defined in terms of the dynamically generated scale $R_{c}$, as it should be.

\subsection{Exact classical $\beta$ function}

To derive an exact equation for the classical $\beta$ function we begin with equation for static scalar field outside an external source

$$
\frac{1}{r^{2}} \frac{d}{d r}\left(r^{2} \frac{d \phi}{d r}\right)-\lambda_{0} \phi^{3}=0
$$

Substituting

$$
\phi=\frac{Q f(r)}{r}
$$

we can rewrite the equation above as

$$
f^{\prime \prime}-f^{\prime}+\alpha_{0} f^{3}=0
$$

where $\alpha_{0}=-\lambda_{0} Q^{2}$ and prime denotes the derivative with respect to $x=\ln \left(r / R_{c}\right)$. Multiplying this equation by $\alpha_{0} f$ and defining running coupling constant as before

$$
\alpha(x)=\alpha_{0} f^{2}(x)
$$

we obtain the following second order differential equation for $\alpha(x)$

$$
\alpha^{\prime \prime}-\frac{\alpha^{\prime 2}}{2 \alpha}-\alpha^{\prime}+2 \alpha^{2}=0 .
$$

Recalling the definition of $\beta$ function, $\beta \equiv \alpha^{\prime}$, and taking into account that

$$
\alpha^{\prime \prime}=\alpha^{\prime} \frac{d \alpha^{\prime}}{d \alpha}=\beta \frac{d \beta}{d \alpha}
$$

equation (4.5) reduces to the first order differential equation:

$$
\beta=2 \alpha^{2}+\frac{1}{2}\left(\frac{d \beta^{2}}{d \alpha}-\frac{\beta^{2}}{\alpha}\right)
$$

which determines the exact classical $\beta$ function. 


\subsection{Weak coupling expansion and renormalons}

Let us first use the exact equation for $\beta$ function to reproduce our perturbative results above. In order to do that we substitute in (4.7)

$$
\beta(\alpha)=\sum_{k=1}^{\infty} \beta_{k} \alpha^{k+1} .
$$

This leads to the following recursion relations for the unknown numerical coefficients $\beta_{k}$

$$
\beta_{1}=2, \quad \beta_{k}=\sum_{m=1}^{k-1}\left(m+\frac{1}{2}\right) \beta_{k-m} \beta_{m} \text { for } k \geq 2,
$$

Using this relations we find

$$
\beta_{1}=2, \beta_{2}=6, \beta_{3}=48, \beta_{4}=570, \beta_{5}=8568, \beta_{6}=151956, \ldots
$$

in complete agreement with (3.11) to an arbitrary order in $\alpha$. Thus we have proven that equation (4.7) yields the exact $\beta$ function which is in complete agreement with the perturbative $\beta$ function.

Let us now find the asymptotic behavior of the perturbative series. One can easily see that for large $k$ the main contribution to the sum in (4.9) comes from the terms with $m=k-1$ and $m=1$ and the recursion relation reduces to

$$
\beta_{k} \simeq(k+1) \beta_{1} \beta_{k-1},
$$

the solution of which is

$$
\beta_{k} \simeq(k+1) ! \beta_{1}^{k}
$$

with $\beta_{1}=2$. Nicely enough this is the same type of factorial behavior we expect in quantum field theory. Moreover, since the coefficient of the factorial is the one-loop $\beta$ function it is natural to identify the origin of this behavior with a renormalon.

In order to clarify the meaning of this renormalon let us consider the perturbative solution of equation (4.7) assuming that $\alpha \ll 1$. Substituting

$$
\beta=2 \alpha^{2}(1+\varepsilon)
$$

in (4.7) we find that $\varepsilon(\alpha)$ satisfies the equation

$$
2 \alpha^{2} \frac{d \varepsilon}{d \alpha}=\frac{\varepsilon}{1+\varepsilon}-3 \alpha(1+\varepsilon)=\varepsilon-3 \alpha+O\left(\varepsilon^{2}, \varepsilon \alpha\right) .
$$

Because both $\varepsilon$ and $\alpha$ are much less than unity we can neglect nonlinear terms. Solving the resulting linear equation one obtains

$$
\varepsilon(\alpha)=\left(\frac{3}{\beta_{1}} \operatorname{Ei}\left(\frac{1}{\beta_{1} \alpha}\right)+C\right) e^{-\frac{1}{\beta_{1} \alpha}}+O\left(\left(e^{-\frac{1}{\beta_{1} \alpha}}\right)^{2}\right),
$$


where $\beta_{1}=2, \operatorname{Ei}(z)$ is the exponential-integral function and $C$ is the constant of integration. Now using the asymptotic expansion of the exponential-integral function at large argument

$$
\operatorname{Ei}(z)=\frac{e^{z}}{z}\left(\sum_{k=0}^{n} \frac{k !}{z^{k}}+O\left(\frac{1}{z^{n+1}}\right)\right),
$$

we find that asymptotically the coefficients of the $\beta$ function in the perturbative expansion grow as $\beta_{k} \sim k ! \beta_{1}^{k}$. This completely clarifies the origin of the renormalon, which is an artifact of the asymptotic expansion of non-analytic function. It also follows from (4.15) that the accuracy of the Borel resummation does not exceed

$$
e^{-\frac{1}{\beta_{1}^{\alpha}}} \sim \frac{r}{R_{c}}
$$

which is the expected non-perturbative uncertainty!

\section{The nonperturbative solution and confinement}

Finally let us use the exact $\beta$ function equation to determine what happens in the infrared region $r>R_{c}$, where the perturbation theory is completely out of the control. Obviously the nonperturbative effective coupling constant can be used to define a static inter-quark potential. Regarding the nature of the classical sources we will leave beyond the scope of paper and concentrate mostly on the calculation of the behavior of the running coupling constant in the infrared region. As we will see this coupling constant determines-similar to QCD- the confining potential between sources. In particular we will compute the energy of an isolated source and a dipole, built out of two opposite charges. In the first case we find the divergent energy which is an indication of confinement and can be interpreted as the absence of isolated sources. The energy of the dipole is finite and positive. Its typical size is of order $R_{c}$ and the binding energy $\sim R_{c}^{-1}$ in case of sources with negligible masses. This is an encouraging hint towards explaining the colorless hadron states in QCD.

\subsection{The infrared coupling constant}

Equation (4.7) for the exact $\beta$ function can rewritten in the form

$$
\frac{d \beta}{d \alpha}=\frac{2 \alpha \beta-4 \alpha^{3}+\beta^{2}}{2 \alpha \beta}
$$

and can be fully investigated using the phase diagram method. The particular solution we need is determined by the perturbative initial condition (3.11). The resulting nonperturbative $\beta$ function, shown in figure 1 , reaches a maximum value about 0.64 for $\alpha \simeq$ 0.69 and after that decreases and vanishes at $\alpha \approx 0.98$. Such behavior of the $\beta$ function in nonperturbative regime is quite nontrivial. The most dramatic effect is the absence of any divergence for the coupling constant, meaning that in case of exact $\beta$ function, which accounts for all resummations, the IR Landau pole is absent. This nonperturbative resummations leads to finite running coupling for any finite $x$ interpolating smoothly between the asymptotically free UV-regime and the IR- region $r \gg R_{c}$. 


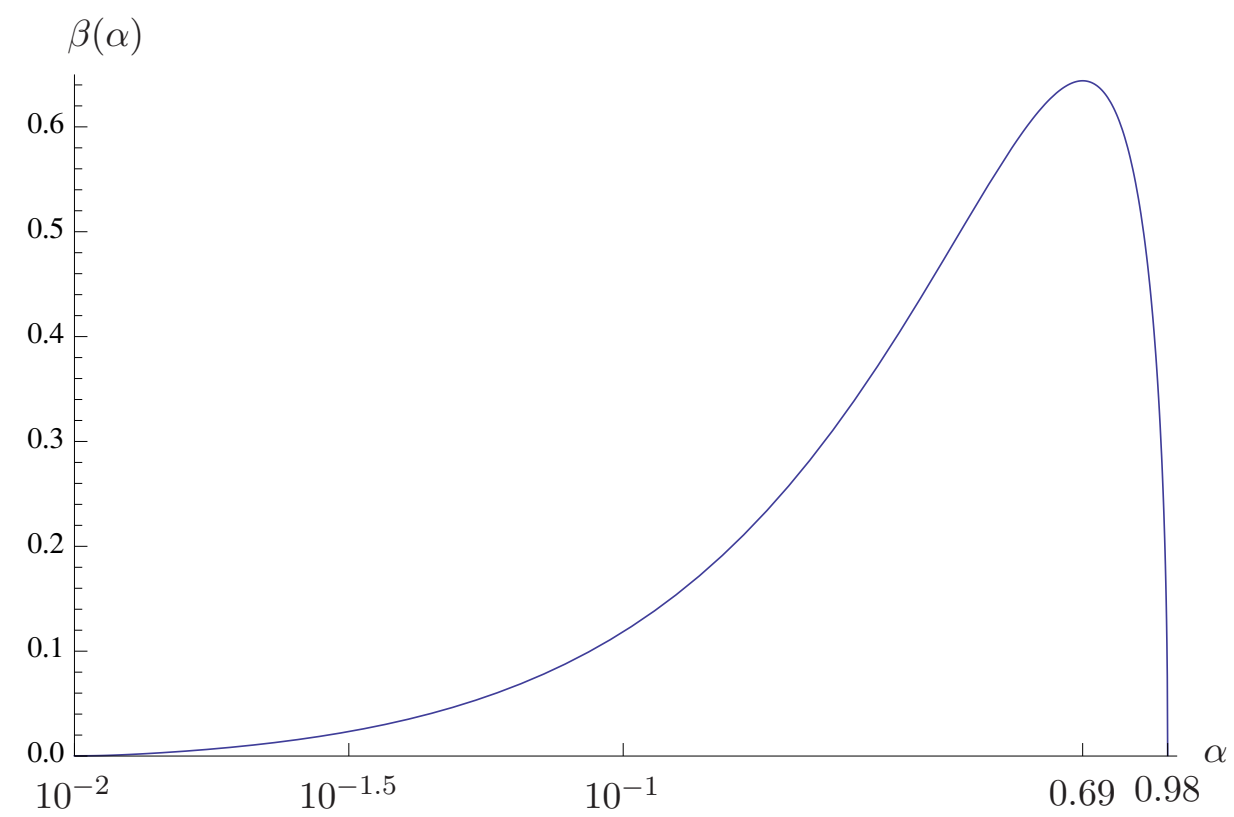

Figure 1. Numerical evaluation of nonperturbative $\beta$ function.

Beyond the first zero of the $\beta$ function it is more convenient to draw $\alpha$ and $\beta$ separately as functions of $x=\ln \left(r / R_{c}\right)$ because both $\alpha$ and $\beta$ become oscillating functions of the scale. In order to find the solution in this region it is more convenient to work directly with equation (4.3) instead of (5.1). This is the equation for a particle "moving" in a positive quartic potential in the presence of negative friction. Such particle "oscillates" and if we neglect for a moment the friction term, the typical "period of oscillation" can be estimated on dimensional grounds as

$$
\Delta x \sim \sqrt{\frac{1}{\alpha_{0} f^{2}}} \sim \sqrt{\frac{1}{\alpha(x)}},
$$

where $f$ is the typical amplitude of oscillations. It is clear that this estimate is valid only if $\Delta x \ll 1$ because otherwise friction term dominates and completely damps the oscillations. However, for $\alpha(x) \gg 1$, when $\Delta x \ll 1$, the friction is not so crucial and the system undergoes oscillations with the amplitude slowly growing due to this negative friction. In order to find how fast this amplitude grows we multiply equation (4.3) by $f$ and rewrite it in the form

$$
\left(f f^{\prime}\right)^{\prime}-f^{\prime 2}-\frac{1}{2}\left(f^{2}\right)^{\prime}+\alpha_{0} f^{4}=0 .
$$

Averaging this equation over the period of oscillations we find

$$
\left\langle f^{\prime 2}\right\rangle=\alpha_{0}\left\langle f^{4}\right\rangle
$$

Multiplying now (4.3) by $f^{\prime}$ leads to the equation

$$
\left(\frac{1}{2} f^{\prime 2}+\frac{1}{4} \alpha_{0} f^{4}\right)^{\prime}=f^{\prime 2}
$$




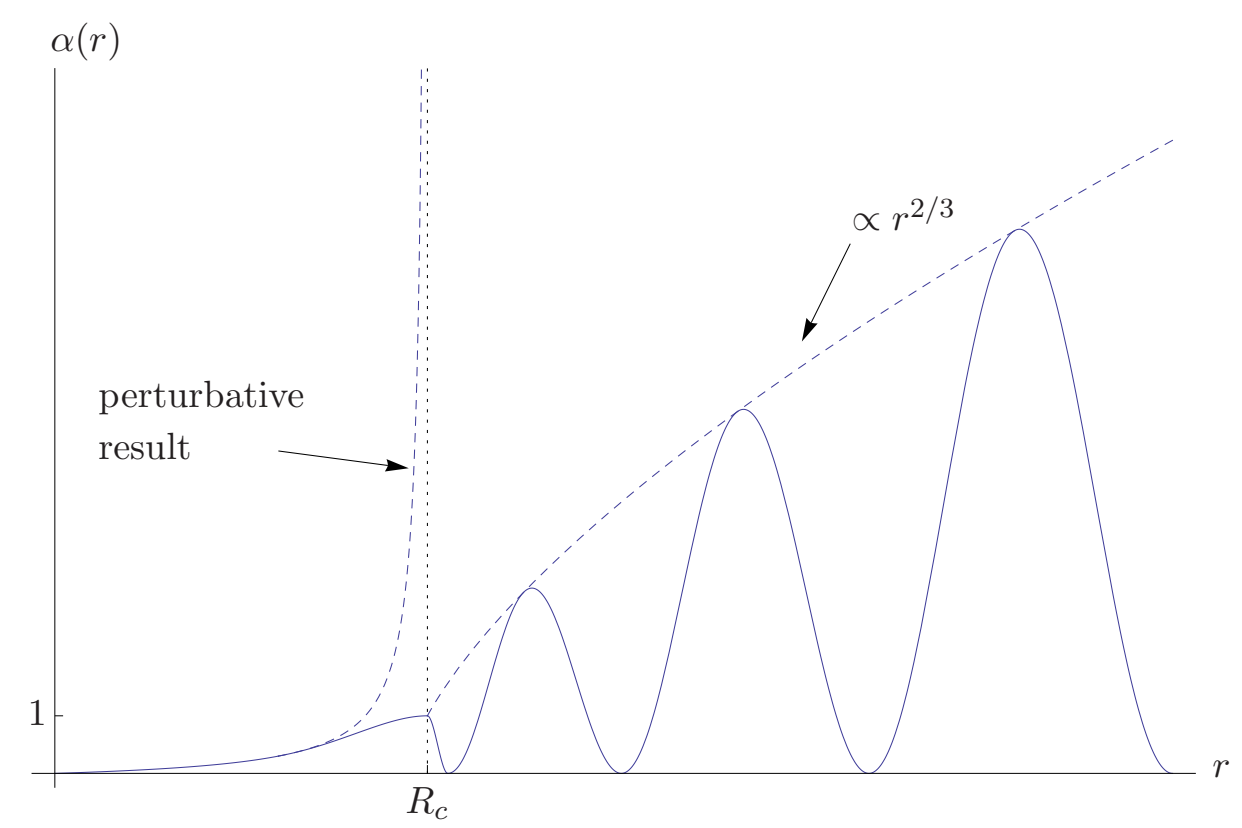

Figure 2. Nonperturbative running coupling.

which after averaging and taking into account (5.3) gives us

$$
\frac{d\left\langle f^{4}\right\rangle}{d x}=\frac{4}{3}\left\langle f^{4}\right\rangle
$$

Finally solving this equation we obtain

$$
\left\langle f^{4}\right\rangle=C \exp \left(\frac{4 x}{3}\right)=C\left(\frac{r}{R_{c}}\right)^{4 / 3}
$$

leading to the following nonperturbative behavior of the running coupling constant

$$
\begin{aligned}
\alpha(r) & =\alpha_{0} f^{2} \simeq \alpha_{0} \sqrt{\left\langle f^{4}\right\rangle} \cos ^{2}\left(\int \sqrt[4]{\alpha_{0}^{2}\left\langle f^{4}\right\rangle} d x\right) \\
& \simeq O(1)\left(\frac{r}{R_{c}}\right)^{2 / 3} \cos ^{2}\left(\frac{r}{R_{c}}\right)^{2 / 3},
\end{aligned}
$$

for $r \gg R_{c}$. In figure 2 we summarize the behavior of the running coupling. As it was said above although according to perturbation theory the coupling constant should become infinite at $r=R_{c}$, in reality this does not happen. The coupling constant remains finite and at scales larger than the confinement scale grows as $r^{2 / 3}$. This is the main non-perturbative result we can extract from the exact $\beta$ function equation.

\subsection{Confinement}

As already discussed we can mimic confinement identifying the classical sources as static quarks and defining a quench approximation to the static inter-quark potential in terms of the effective coupling in the IR region. 
Since in our case the self-interaction contribution to the energy goes like $\phi^{4} \sim f^{4} \sim$ $\alpha(r)^{2}$ we can define the static potential as:

$$
V(r) \sim \frac{\alpha^{2}(r)}{r}
$$

Using the non-perturbative value of the running coupling (5.7) we get

$$
V(r) \sim O(1) R_{c}^{-1}\left(\frac{r}{R_{c}}\right)^{1 / 3}
$$

To check this qualitative result let us compute the energy of the field created by a static external charge. Since the field is static and spherically symmetric, the total energy is given by the expression

$$
\begin{aligned}
E & =\frac{1}{2} \int\left((\nabla \phi)^{2}+\frac{\lambda_{0}}{2} \phi^{4}\right) d^{3} x=2 \pi \int\left(\left(\partial_{r} \phi\right)^{2}+\frac{\lambda_{0}}{2} \phi^{4}\right) r^{2} d r \\
& =2 \pi Q^{2} \int\left(\left(r \partial_{r} f-f\right)^{2}-\frac{1}{2} \alpha_{0} f^{4}\right) \frac{d r}{r^{2}}
\end{aligned}
$$

Note that although the contribution of the second term is negative for negative $\lambda_{0}$, the total energy is positive because the gradient term dominates. The integral above diverges when $r \rightarrow 0$. This divergence has an ultraviolet origin and it is the same as well known divergence of the self-energy of classical point-like electric charge. Therefore it can be removed using standard methods. We will focus instead on the IR contribution to the energy. Taking into account that at $r \gg R_{c}$,

$$
\left\langle\left(r \partial_{r} f\right)^{2}\right\rangle=\left\langle f^{\prime 2}\right\rangle=\alpha_{0}\left\langle f^{4}\right\rangle,
$$

the following expression for the infrared contribution to the total energy is obtained

$$
E \simeq \pi Q^{2} \int_{R_{c}}^{r} \alpha_{0}\left\langle f^{4}\right\rangle \frac{d r}{r^{2}} \sim O(1) Q^{2} \frac{1}{R_{c}}\left(\frac{r}{R_{c}}\right)^{1 / 3},
$$

in agreement with the qualitative expectations. The energy of the isolated charge diverges as $r^{1 / 3}$ and therefore it cannot exist as a free asymptotic state. This can be interpreted as a hint of confinement of isolated sources.

One can also build "colorless configuration" using two opposite charges $Q$ and $-Q$ separated by distance $l$. At distances $r \gg l$, the field $\phi$ decreases as $r^{-2}$ and equation (2.3) becomes

$$
\frac{1}{r^{2}} \frac{d}{d r}\left(r^{2} \frac{d \phi}{d r}\right)=-4 \pi Q \delta(\mathbf{x})+\lambda_{0} \frac{Q^{3} l^{3}}{r^{6}}
$$

Since in this case the anti-screening effect, determined by the last term in this equation, is completely irrelevant at large distances we conclude that the total energy of the dipole system is infrared convergent. When the distance between charges exceeds the confinement scale $R_{c}$ the infrared contribution of the scalar field becomes essential and the total energy is

$$
E \sim O(1) R_{c}^{-1}\left(\frac{l}{R_{c}}\right)^{1 / 3}
$$


Hence, the interaction potential between two charges grows as distance in power one third. This can be interpreted as a confining potential leading to a natural estimate for the mass scale of the dipole configuration to be of order $m \sim O(1) R_{c}^{-1}$.

\section{Discussion and speculations}

We have shown that certain essential properties of the quantum field theory usually considered as having quantum origin can be revealed already at the classical level. In particular, the renormalization group structure of the theory including the phenomenon of dimensional transmutation is already encoded in the classical equations.

So far we have considered only the self-interacting scalar field with negative coupling constant and external sources. One can naturally ask up to what extent the qualitative results obtained in this paper are useful in application to gauge theories, such as QCD. An encouraging sign, that classical RG treatment can be generalized for such theories is provided by the following simple scaling argument. As we have found, the logarithmic effect of anti-screening comes from the term of $\phi^{3}$ in the equation for the scalar field. In the perturbation theory this term represents the density of the charge induced by selfinteraction and it drops as $r^{-3}$ as distance $r$ grows. In QCD the gauge field equations for gluons contain two kinds of self-interaction terms which drop in a similar way, namely, $A^{3}$ and $A \partial A$. Only $A \partial A$ gives the negative contribution to the $\beta$ function. This term leads to anti-screening effect inducing the density of the colored charge decaying as $r^{-3}$ similar to the case of scalar field. Because the structure of the self-interaction terms is different (in one case it is $\phi^{4}$ and in the other $A^{2} \partial A$ ) the interaction potential between two sources in gauge theories can grow with the distance not necessarily as $r^{1 / 3}$, but as $r^{\alpha}$, where $0<\alpha \leq 1$. So, the linear growth is not excluded. However, the linear growth, although leading to confined charges does not necessarily imply the formation of QCD flux tube (see appendix B).

\section{A On the triviality of $\lambda \phi^{4}$ theory with positive $\lambda$}

We can use the exact $\beta$ function equation to check the triviality of $\lambda_{0} \phi^{4}$ theory in the case of positive $\lambda_{0}$ in four dimensions (this triviality was rigorously proved in five and higher dimensions in $[19,20])$. In the case of positive $\lambda_{0}$ it is convenient to change the signs in the definitions of $\alpha_{0}$ and $x$, so that,

$$
\alpha_{0} \equiv \lambda_{0} Q^{2}>0, \quad x \equiv \ln \left(r_{0} / r\right) .
$$

In this case the $\beta$ function defined in (3.10) is related to the standard $\beta_{s t}$ function used in the literature as $\beta_{s t}=\beta / 2$. With these redefinitions the equation for the exact $\beta$ function is obtained from (4.7) by substituting $\beta \rightarrow \beta$ and $\alpha \rightarrow-\alpha$ :

$$
\beta=2 \alpha^{2}-\frac{1}{2}\left(\frac{d \beta^{2}}{d \alpha}-\frac{\beta^{2}}{\alpha}\right) .
$$

For $\alpha \ll 1$ the perturbative solution of this equation is

$$
\beta=2 \alpha^{2}-6 \alpha^{3}+48 \alpha^{4}-570 \alpha^{5}+\ldots
$$


The Gell-Mann-Low equation to one loop,

$$
\frac{d \alpha(x)}{d x}=2 \alpha^{2}(x)
$$

gives us

$$
\alpha(r)=\frac{\alpha_{0}}{1-2 \alpha_{0} x}=\frac{\lambda_{0} Q^{2}}{1-2 \lambda_{0} Q^{2} \ln \left(r_{0} / r\right)} .
$$

According to this result the coupling constant blows up at the Landau pole

$$
r_{L}=r_{0} e^{-\frac{1}{2 \lambda_{0} Q^{2}}} .
$$

The essence of the proof of $\lambda \phi^{4}$ triviality can be reduced to showing that this UV pole will survives at nonperturbative level. This is not so obvious because as we have seen the IR one loop Landau pole (for negative $\lambda_{0}$ ) disappears after resummation of the perturbative expansion. To find out whether the pole survives or not for positive $\lambda$, let us solve equation (A.2) in strong coupling regime, $\alpha \gg 1$. We will do it perturbatively in terms of the inverse powers of $\alpha$. Neglecting the linear $\beta$ term in (A.2) we have

$$
\frac{d \beta^{2}}{d \alpha}-\frac{\beta^{2}}{\alpha} \simeq 4 \alpha^{2}
$$

and the corresponding solution of this equation is

$$
\beta=\sqrt{2} \alpha^{3 / 2}
$$

Rewriting (A.2) as

$$
\frac{d \beta^{2}}{d \alpha}-\frac{\beta^{2}}{\alpha}=4 \alpha^{2}-2 \beta
$$

substituting in the right hand side of this equation the result (A.8) and solving the obtained inhomogeneous linear equation for $\beta^{2}$ we obtain

$$
\beta=\sqrt{2} \alpha^{3 / 2}\left(1-\frac{2 \sqrt{2}}{3} \alpha^{-1 / 2}\right)^{1 / 2}=\sqrt{2} \alpha^{3 / 2}\left(1-\frac{\sqrt{2}}{3} \frac{1}{\sqrt{\alpha}}+O\left(\left(\frac{1}{\sqrt{\alpha}}\right)^{2}\right)\right)
$$

This procedure can be repeated recursively giving us higher order power corrections in the expansion in $1 / \sqrt{\alpha} \ll 1$. Thus we see that for very large $\alpha$ the solution (A.8) becomes more and more accurate and the behavior of $\beta$ functions confirms the expectations in $[17,18]$. The solution (A.8) exactly matches the one loop result in (A.3) at $\alpha=1 / 2$. Using this to fix the integration constant in the Gell-Mann-Low equation

$$
\frac{d \alpha(x)}{d x}=\sqrt{2} \alpha^{3 / 2}
$$

we obtain the following non-perturbative result valid for $\alpha \gg 1$

$$
\alpha(r)=8\left(\frac{\alpha_{0}}{1-2 \alpha_{0}(x-1)}\right)^{2}=8\left(\frac{\lambda_{0} Q^{2}}{1-2 \lambda_{0} Q^{2} \ln \left(r_{0} / r e\right)}\right)^{2}
$$

Thus we see that even after complete resummation of the perturbative expansion the Landau pole survives and its non-perturbative location is at (figure 3):

$$
r_{L}=\frac{r_{0}}{e} e^{-\frac{1}{2 \lambda_{0} Q^{2}}}
$$

This leads to the triviality of $\lambda \phi^{4}$ theory. 


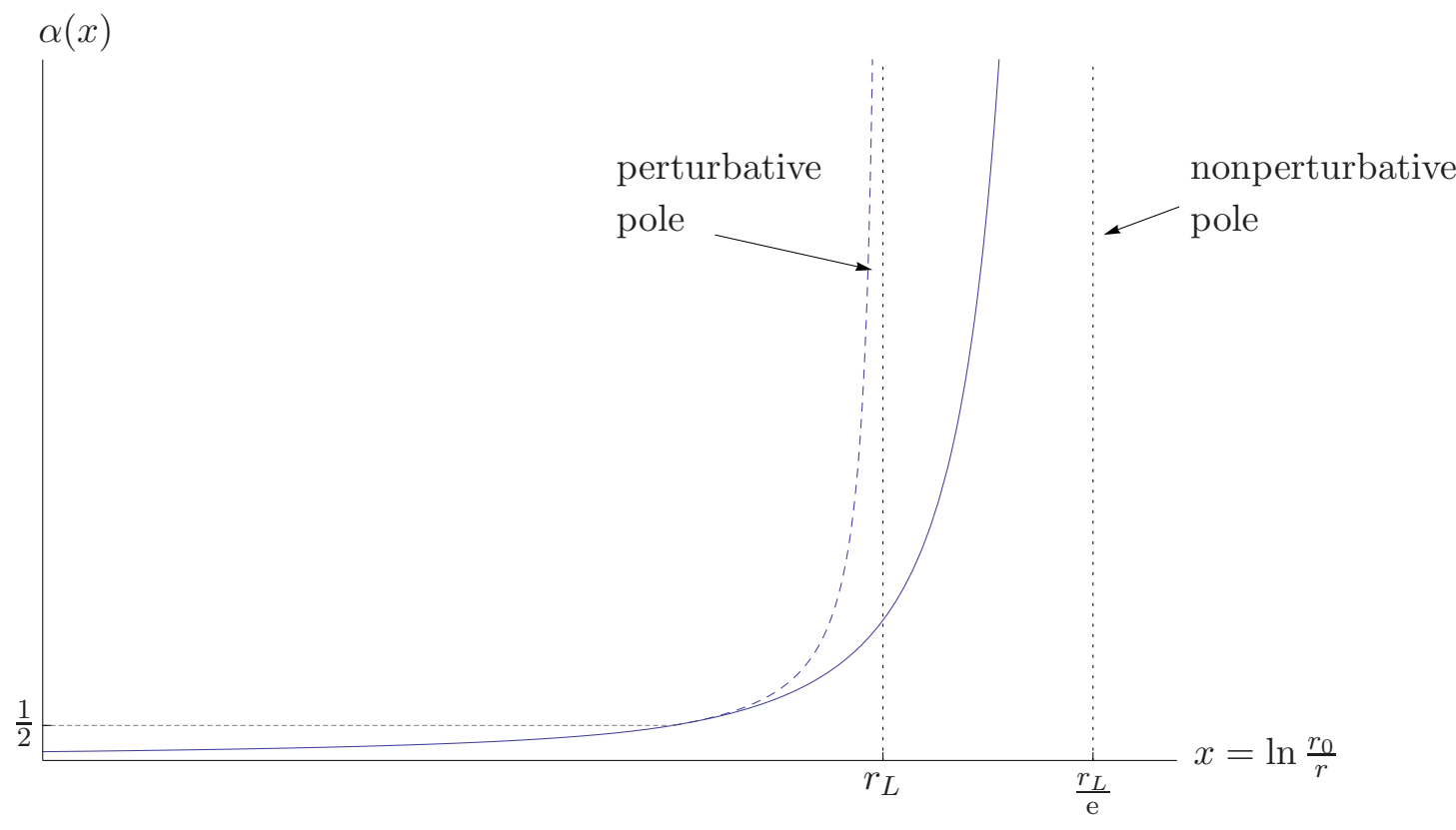

Figure 3. Landau pole.

\section{B Confinement with and without strings}

As we have shown in the paper the potential between the external sources grows unbounded with the separation as a third power of it. In such a picture, charge and anti-charge are confined but the confinement in not due to formation of a flux tube (string-type object) but rather due to formation of a finite energy dipole. One may think, that this is a peculiarity of not having a linear growth of the potential. In this appendix we will discuss this issue and show that even the linear potential does not necessarily imply the existence of a string.

To demonstrate this we begin with an $\mathrm{SO}(3)$ sigma model of an isotriplet scalar field $\phi^{a}(a=1,2,3)$ with Lagrangian:

$$
\mathcal{L}=\partial_{\mu} \phi^{a} \partial^{\mu} \phi^{a}-\lambda^{2}\left(\phi^{a} \phi^{a}-v^{2}\right)^{2} .
$$

In this case the equation of motion has the static spherically-symmetric solution (see e.g. [21])

$$
\phi^{a}=f(r) \frac{x^{a}}{r},
$$

where $x^{a}$ are Cartesian space coordinates and $f(r)$ is the function with the following asymptotic properties

$$
f(0)=0,\left.\quad f(r)\right|_{r \gg(\lambda v)^{-1}} \rightarrow v
$$

The size of the region where $f(r)$ is different from $v$ is of order $r_{c}=(\lambda v)^{-1}$. The solution above is the 't Hooft - Polyakov monopole in the limit of zero gauge coupling. Because in this limit the gauge fields become massless and decouple from $\phi$ this solution is often referred to as a global magnetic monopole.

The energy of this monopole can be easily estimated by considering separately the contribution from core $\left(r<r_{c}\right)$ and from the rest. The core contribution is of order $v$, and 
can be neglected compared to the energy in the region $r>r_{c}$, where $f(r)$ can be set to be equal $v$. Then the contribution of the gradients of the angles (Nambu-Goldstone modes) is divergent and we have to cut-off the integral at some $R \gg r_{c}$. The resulting energy of the isolated "charge",

$$
E_{r>r_{c}} \simeq 4 \pi \int_{r_{c}}^{R} d r v^{2} \simeq 4 \pi R v^{2}
$$

is linearly divergent. This implies that the potential between two opposite charges is linear. In fact, let us consider an anti-monopole placed at distance $R$ from the monopole. The effect of anti-monopole is to cut the divergent integral at $r=R$ and the resulting potential is

$$
V_{R>r_{c}} \simeq R / r_{c}^{2}
$$

thus confining monopole-antimonopole configuration! This picture is very different from the QCD flux-tube (string) confinement. To understand this difference let us confront them considering heavy quark-anti-quark pair placed at distance $R$ apart. In the absence of light quarks this distance $R$ can be much larger than the QCD scale, $r_{Q C D} \equiv \Lambda_{Q C D}^{-1}$. In string picture the force between this pair is mediated by a stretched string (electric flux tube) of constant tension $\sim \Lambda_{Q C D}^{2}$, giving the potential

$$
V_{R>r_{Q C D}} \simeq R / r_{Q C D}^{2}
$$

similar to the monopole-anti-monopole potential. However, in the monopole case the flux is not confined to a string and for monopole-anti-monopole it has a dipole configuration (see figure 4). As a result the "monopole color" is not bounded within the string of width $r_{c}$ and can be probed everywhere in the space around the monopole at $r>r_{c}$. On the other hand, in the case of string the only possibility to probe the color of the charge is to penetrate within the string of width $r_{Q C D}$.

The difference between these two pictures can be stressed even more if we notice that the theory (B.1) allows to be "deformed" to the theory in which true strings connecting monopoles appear. The appearance of "open-color" monopole-antimonopole configuration is due to $S(3) / \mathrm{U}(1)$ topology of the vacuum manifold with nontrivial $\pi_{2}$. To change this topology we can further deform the vacuum manifold by spontaneous breaking of the remaining $\mathrm{U}(1)$ symmetry. This can be done by introducing additional scalar field $\chi_{\alpha}$ $(\alpha=1,2)$ in a doublet representation of the $\mathrm{SO}(3)$ group. The Lagrangian then becomes

$$
\mathcal{L}=\partial_{\mu} \phi \partial^{\mu} \phi+\partial_{\mu} \chi^{*} \partial^{\mu} \chi-\lambda^{2}\left(\phi^{2}-v^{2}\right)^{2}-\lambda_{1}^{2}\left(\chi^{*} \chi-v_{1}^{2}\right)^{2}+h \chi^{*} \phi \chi+h^{\prime *} \phi^{2} \chi,
$$

where the contraction of indices is obvious. The parameters $\left(\lambda, \lambda_{1}, v, v_{1}, h, h^{\prime}\right)$ are chosen in such a way that the field $\chi$ develops an expectation value $v^{\prime} \ll v$. In this limit, core of the monopole remains nearly unchanged. However at very large distances the field $\chi$ dramatically changes the monopole field. The presence of the second field with nonzero expectation value leads to the following hierarchical symmetry-breaking pattern,

$$
\mathrm{SO}(3) \rightarrow \mathrm{U}(1) \rightarrow 1
$$



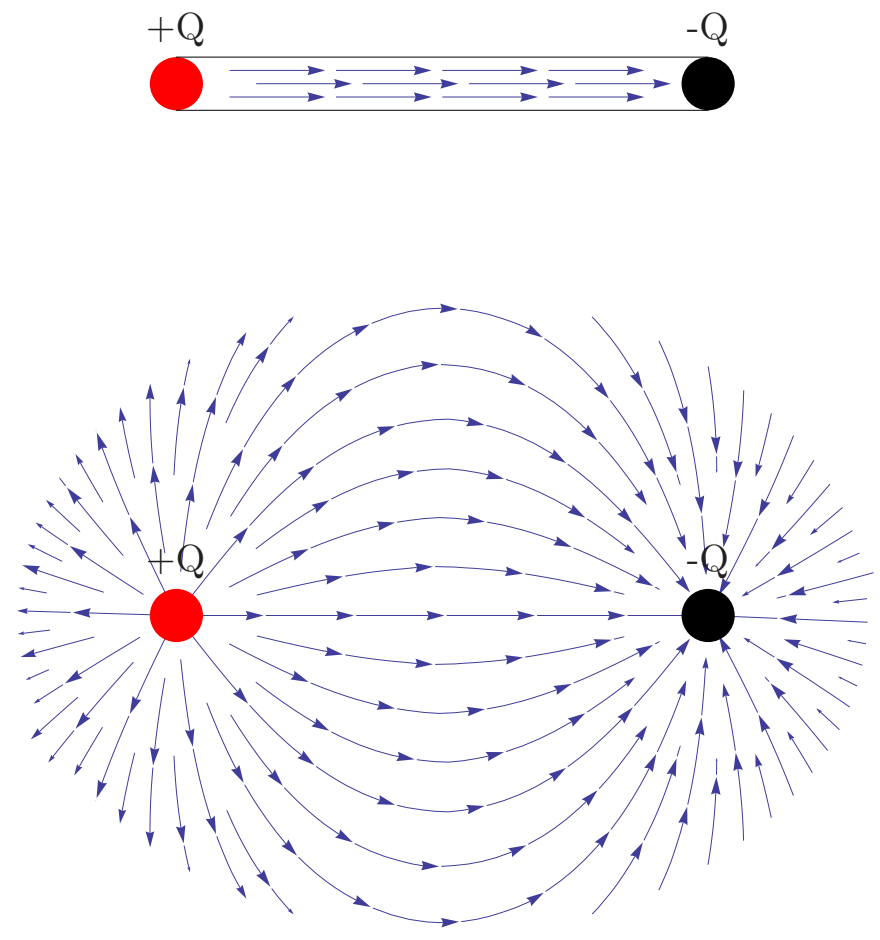

Figure 4. String vs. dipole.

and the vacuum manifold becomes topologically-trivial. As a result, the static isolated monopoles can not exist anymore. However, due the hierarchy of symmetry breaking, $v \gg v^{\prime}$, monopoles do not simply disappear from the spectrum, but rather get connected by the strings. Either strings or monopoles are stable in two limits: $v=\infty$ and finite $v^{\prime}$ or $v$ finite and $v^{\prime}=0$, respectively. However, when both $v$ and $v^{\prime}$ are finite, they can only exist as hybrid configuration, namely, monopoles connected by strings. This picture is more close to the usual string confinement because here the monopole magnetic flux gets confined into the string. The thickness of this string is $\sim 1 / v^{\prime} \gg r_{c}$, and its tension is $v^{\prime 2} \ln L$, where $L$ is the string's length. In other words, the tension of the string is logarithmically divergent. For example, for the string oriented in $z$ direction the field $\chi$ near the string but far away from the monopoles, is

$$
\chi_{\alpha} \simeq \delta_{\alpha}^{1} f(\rho) e^{i \theta}
$$

where $f(\rho)$ vanishes at $\rho=0$ and approaches constant for $\rho>1 / v^{\prime}$ in the cylindric coordinates $\rho, \theta$. The energy of this configuration is logarithmically divergent with the natural cut-off scale of order string size.

The picture above can be summarized as follows. For $R \ll 1 / v^{\prime}$, the potential between monopoles is linear and field configuration is of dipole type, but for $R \gg 1 / v^{\prime}$ the flux is not spread anymore and becomes confined by a string. This leads to the modification of 
the potential (B.5), which becomes

$$
V(R) \sim v^{\prime 2} R \ln R .
$$

This is not such a dramatic change in the potential, but more important is the qualitative change of the physical picture, because now the monopoles are becoming confined by the string. The consideration above illustrates a very important point, that the confinement can have very distinct physical origin for the same growing potential between charges.

Finally, we will consider here one more theory, where confining potential is due to usual electric flux. Let us consider U(1) theory with Lagrangian

$$
L=\left(F_{\mu \nu} F^{\mu \nu}\right)^{\alpha}+A_{\mu} j^{\mu} .
$$

Unlike (B.1), which describes healthy theory, the legitimacy of this theory as a quantum field theory of $U(1)$ gauge field is much less obvious. However, since we are interested only in geometric properties of the classical electric fluxes, we will use it for our purposes. In this case the equations of motion are

$$
\partial^{\mu}\left(2 \alpha\left(F^{2}\right)^{\alpha-1} F_{\mu \nu}\right)=j_{\nu} .
$$

For the static charge $j_{\mu}=\delta_{\mu}^{0} \delta(r) Q$, which produces spherically-symmetric electric field,

$$
F_{j 0} \equiv E_{j}(r)=E(r) \frac{x_{j}}{r},
$$

they become,

$$
\partial^{j}\left(2 \alpha E(r)^{2 \alpha-2} E_{j}(r)\right)=\delta(r) Q .
$$

It immediately follows from here that

$$
2 \alpha E(r)^{2 \alpha-1}=\frac{Q}{r^{2}},
$$

and hence

$$
E(r)=\left(\frac{Q}{2 \alpha r^{2}}\right)^{\frac{1}{2 \alpha-1}} .
$$

The energy of an isolated charge smeared over a sphere $r_{0}$ diverges as

$$
E_{\text {charge }}=\int_{r_{0}}^{R} r^{2} d r\left(\frac{Q}{2 \alpha r^{2}}\right)^{\frac{2 \alpha}{2 \alpha-1}} \sim Q^{\frac{2 \alpha}{2 \alpha-1}} R^{\frac{2 \alpha-3}{2 \alpha-1}},
$$

for either $\alpha>3 / 2$ or $\alpha<1 / 2$ when exponent is positive. Thus, in both these cases the energy of an isolated charge diverges. However, in this case the finite energy of the chargeanticharge configuration is not for granted automatically! The situation is much more subtle than in the usual case because of very strong non-linearity, which make superposition principle not applicable to $E_{j}$. However, the superposition principle in this case is valid for $E(r)^{2 \alpha-2} E_{j}(r)$. Therefore, the electric field of the dipole of size $D$ is given by,

$$
E(r)_{D} \simeq\left(\frac{Q D}{2 \alpha r^{3}}\right)^{\frac{1}{2 \alpha-1}} .
$$

The corresponding energy is

$$
E_{\text {charge }} \sim(D Q)^{\frac{2 \alpha}{2 \alpha-1}} R^{\frac{-3}{2 \alpha-1}},
$$

and it is finite only for $\alpha>3 / 2$. 


\section{Acknowledgments}

We are grateful to L. Alvarez-Gaume, C. Bachas, A. Barvinski, M. Henneaux and I. Sachs for discussions and valuable comments. We would like to thank T. Hofbaur for the help with preparing figures.

The work of G.D. was supported in part by Humboldt Foundation under Alexander von Humboldt Professorship, by European Commission under the ERC advanced grant 226371, by David and Lucile Packard Foundation Fellowship for Science and Engineering and by the NSF grant PHY-0758032. The work of C.G. was supported in part by Grants: FPA 2009-07908, CPAN (CSD2007-00042) and HEPHACOS P-ESP00346. V.M. is supported by TRR 33 "The Dark Universe" and the Cluster of Excellence EXC 153 "Origin and Structure of the Universe".

Open Access. This article is distributed under the terms of the Creative Commons Attribution Noncommercial License which permits any noncommercial use, distribution, and reproduction in any medium, provided the original author(s) and source are credited.

\section{References}

[1] D. Gross and F. Wilczek, Ultraviolet behavior of non-Abelian gauge theories, Phys. Rev. Lett. 30 (1973) 1343 [INSPIRE].

[2] H. Politzer, Reliable perturbative results for strong interactions?, Phys. Rev. Lett. 30 (1973) 1346 [InSPIRE].

[3] K. Symanzik, A field theory with computable large-momenta behavior, Lett. Nuovo Cim. 6S2 (1973) 77 [INSPIRE].

[4] G. Parisi, Deep inelastic scattering in a field theory with computable large-momenta behaviour, Lett. Nuovo Cim. 7S2 (1973) 84 [INSPIRE].

[5] K. Wilson, RG and strong interactions, Phys. Rev. D 3 (1971) 1818.

[6] K. Wilson and J. Kogut, The renormalization group and the $\varepsilon$-expansion, Phys. Rept. 12 (1974) 75.

[7] G. 't Hooft, Dimensional regularization and the renormalization group, Nucl. Phys. B 61 (1973) 455 [inSPIRE].

[8] G. 't Hooft, The renormalization group and Quantum Field Theory, lecture at Eight graduate school, Doorwerth The Netherlands January 25-29 1988.

[9] M. Gell-Mann and F. Low, Quantum electrodynamics at small distances, Phys. Rev. 95 (1954) 1300 [inSPIRE].

[10] L.D. Landau, A.A. Abrikosov and I.M. Khalatnikov, On the removal of infinities in quantum electrodynamics, Dokl. Akad. Nauk Ser. Fiz. 95 (1954) 497.

[11] L.D. Landau, A.A. Abrikosov and I.M. Khalatnikov, Asymptotic expression of the Green's function of the electron in quantum electrodynamics, Dokl. Akad. Nauk Ser. Fiz. 95 (1954) 773 .

[12] L.D. Landau, A.A. Abrikosov and I.M. Khalatnikov, An asymptotic expression for the Green function of a photon in quantum electrodynamics, Dokl. Akad. Nauk Ser. Fiz. 95 (1954) 1177. 
[13] L. Lipatov, Divergence of the perturbation theory series and the quasiclassical theory, Sov. Phys. JETP 45 (1977) 216 [Zh. Eksp. Teor. Fiz. 72 (1977) 411] [InSPIRE].

[14] E. Brézin, J. Le Guillou and J. Zinn-Justin, Perturbation theory at large order. 1. The $\phi^{2 N}$ interaction, Phys. Rev. D 15 (1977) 1544 [INSPIRE].

[15] E. Brézin, J.-C. Le Guillou and J. Zinn-Justin, Perturbation theory at large order. 2. Role of the vacuum instability, Phys. Rev. D 15 (1977) 1558 [INSPIRE].

[16] G. Parisi, Asymptotic estimates in perturbation theory with fermions, Phys. Lett. B 66 (1977) 382 [INSPIRE].

[17] L.D. Landau and I. Pomeranchuk, On point interactions in quantum electrodynamics, Dokl. Akad. Nauk Ser. Fiz. 102 (1955) 489 [INSPIRE].

[18] I. Pomeranchuk, Vanishing of renormalized charge in quantum electrodynamics, Dokl. Akad. Nauk Ser. Fiz. 103 (1955) 1005.

[19] M. Aizenman, Geometric analysis of $\phi^{4}$ fields and Ising models (parts 1 \& 2), Commun. Math. Phys. 86 (1982) 1 [INSPIRE].

[20] J. Fröhlich, On the triviality of $\Lambda\left(\phi^{4}\right)$ in D-dimensions theories and the approach to the critical point in $D_{>} 4$ dimensions, Nucl. Phys. B 200 (1982) 281 [INSPIRE].

[21] A. Vilenkin and E.P.S. Shellard, Cosmic strings and other topological defects, Cambridge University Press, Cambridge U.K. (1994). 